\title{
CELL-LIKE MAPPINGS OF HILBERT CUBE MANIFOLDS : APPLICATIONS TO SIMPLE HOMOTOPY THEORY
}

\author{
BY T. A. CHAPMAN ${ }^{1}$ \\ Communicated by R. D. Anderson, March 19, 1973
}

\begin{abstract}
In this note an infinite-dimensional result is established which implies the following finite-dimensional result as a special case: If $K, L$ are finite $C W$-complexes and $f$ is a map of $K$ onto $L$ such that each point-inverse has trivial shape, then $f$ is a simple homotopy equivalence.
\end{abstract}

1. Introduction. A Hilbert cube manifold, or $Q$-manifold, is a separable metric manifold modeled on the Hilbert cube $Q$. A mapping $f: X \rightarrow Y$ is said to be $C E$, or cell-like, provided that $f$ is onto, proper (i.e. the inverse image of each compactum is compact), and each point-inverse $f^{-1}(y)$ has trivial shape (in the sense of Borsuk [1]). Here is the main result of this note.

THEOREM 1. If $X, Y$ are $Q$-manifolds and $f: X \rightarrow Y$ is $a$ CE mapping, then $f$ is proper homotopic to a homeomorphism of $X$ onto $Y$.

The key technical result needed for the proof of Theorem 1 is the solution of an infinite-dimensional $C E$ handle problem, which is stated in Lemma 2 here and is the main result of [7]. The proof of Lemma 2 uses a considerable amount of infinite-dimensional topology along with the torus technique of $[10]$, which was crucial in establishing a corresponding finitedimensional result.

A CW-complex is strongly locally-finite provided that it is the union of a countable, locally-finite collection of finite subcomplexes. The following is an application of Theorem 1 to infinite simple homotopy equivalences of strongly locally-finite $\mathrm{CW}$-complexes (see [9] for a definition of an infinite simple homotopy equivalence).

THEOREM 2. If $K, L$ are strongly locally-finite $\mathrm{CW}$-complexes and $f$ : $K \rightarrow L$ is a CE mapping, then $f$ is an infinite simple homotopy equivalence.

This generalizes a result of the author's [6], where it was shown that any homeomorphism between strongly locally-finite CW-complexes is an infinite simple homotopy equivalence. We remark that Cohen [8] had

AMS (MOS) subject classifications (1970). Primary 57C10; Secondary 58B05.

Key words and phrases. Hilbert cube manifold, $C E$ mapping, infinite simple homotopy equivalence.

${ }^{1}$ Supported in part by NSF Grant GP-28374. 
previously established a version of Theorem 2 for $C E$ mappings of finite simplicial complexes in which the mappings are $P L$. More recently R. D. Edwards has proved a version of Theorem 2 for arbitrary $C E$ mappings of countable, locally-finite, simplicial complexes.

2. Some lemmas. If $f, g: X \rightarrow Y$ are maps and $\mathscr{U}$ is an open cover of $Y$, then we say that $f$ is $\mathscr{U}$-close to $g$ provided that for each $x \in X$ there exists a $U \in \mathscr{U}$ containing both $f(x)$ and $g(x)$. If $A \subset Y$, then we say that $f=g$ over $A$ provided that $f^{-1}(A)=g^{-1}(A)$ and $f\left|f^{-1}(A)=g\right| g^{-1}(A)$. We also say that $f$ is $1-1$ over $A$ provided that $f \mid f^{-1}(A)$ is $1-1$.

If $X$ is a $Q$-manifold, then a closed subset $A$ of $X$ is a $Z$-set in $X$ provided that given any nonnull and contractible open subset $U$ of $X, U \backslash A$ is also nonnull and contractible. The following result is established in [7].

LEMMA 1. Let $X$ and $Y$ be $Q$-manifolds, $f: X \rightarrow Y$ be a $C E$ mapping, and let $A \subset Y$ be a $Z$-set in $Y$. If $\mathscr{U}$ is an open cover of $Y$, then there exists a CE mapping $g: X \rightarrow Y$ such that $g$ is 1-1 over $A, g^{-1}(A)$ is a Z-set in $X$, and $g$ is $\mathscr{U}$-close to $f$.

For notation for the next result let $R^{n}$ denote Euclidean $n$-space (where $R^{1}=R$ ) and let $B_{r}^{n}$ denote the standard $n$-ball of radius $r$, with interior $\operatorname{Int}\left(B_{r}^{n}\right)$ and boundary $\operatorname{Bd}\left(B_{r}^{n}\right)=S_{r}^{n-1}$. The following is the CE handle result of [7].

LeMma 2. Let $X$ be a $Q$-manifold and let $f: X \rightarrow B_{1}^{k} \times R^{n} \times Q$ be a CE mapping, for $k \geqq 0$ and $n \geqq 1$, such that $f$ is 1-1 over $S_{1}^{k-1} \times R^{n} \times Q$ and $f^{-1}\left(S_{1}^{k-1} \times R^{n} \times Q\right)$ is a Z-set in $X$. Then there exists a CE mapping $g: X \rightarrow B_{1}^{k} \times R^{n} \times Q$ such that $g=f$ over $\left(S_{1}^{k-1} \times R^{n} \times Q\right) \cup\left(B_{1}^{k} \times\right.$ $\left.\left(R^{n} \backslash \operatorname{Int}\left(B_{2}^{n}\right)\right) \times Q\right)$ and $g$ is 1-1 over $B_{1}^{k} \times B_{1}^{n} \times Q$.

We now use Lemmas 1 and 2 to prove the following result which will be needed in the proof of Theorem 1 .

Lemma 3. Let $X$ and $Y$ be $Q$-manifolds, $f: X \rightarrow Y$ be a $C E$ mapping, $K$ be a finite simplicial complex, and let $\varphi: K \times Q \times R \rightarrow Y$ be an open embedding. Then there exists $C E$ mapping $g: X \rightarrow Y$ such that $g=f$ over $Y \backslash \varphi(K \times Q \times(-2,2)), g$ is 1-1 over $\varphi(K \times Q \times[-1,1])$, and

$$
g \simeq f \operatorname{rel} X \backslash f^{-1} \varphi(K \times Q \times(-2,2))
$$

Proof. By taking a regular neighborhood of $K$ in an Euclidean space we get a compact, combinatorial, $n$-manifold $M$ which is simple homotopy equivalent to $K$. The main result of [11] asserts that if $A$ and $B$ are simple homotopy equivalent finite simplicial complexes, then $A \times Q$ is homeomorphic to $B \times Q$. Thus no generality is lost by replacing $K$ with a compact, combinatorial, $n$-manifold $M$. 
By choosing a $P L$ handle decomposition of $M$ we can write $M=$ $M_{-1} \cup M_{0} \cup \cdots \cup M_{n}$, where $M_{-1}$ is a regular neighborhood of $\partial M$ and each $M_{i}$ is a compact $P L$ submanifold of $M$ which is obtained from $M_{i-1}$ by adding handles of index $i$. For each $i,-1 \leqq i \leqq n$, we will show how to construct a $C E$ mapping $g_{i}: X \rightarrow Y$ such that $g_{i}=f$ over $Y \backslash \varphi(M \times Q \times(-2,2)), g_{i}$ is 1-1 over a neighborhood of

$$
\varphi\left(M_{i} \times Q \times[-1,1]\right),
$$

and $g_{i} \simeq f \operatorname{rel} X \backslash f^{-1} \varphi(M \times Q \times(-2,2))$.

For the construction of $g_{-1}$ we first note that $\varphi(\partial M \times Q \times[-1,1])$ is a $Z$-set in the $Q$-manifold $\varphi(M \times Q \times(-2,2))$. Applying Lemma 1 to the restricted $C E$ mapping

$$
f \mid: f^{-1} \varphi(M \times Q \times(-2,2)) \rightarrow \varphi(M \times Q \times(-2,2))
$$

we can find a $C E$ mapping $g_{-1}^{\prime}: f^{-1} \varphi(M \times Q \times(-2,2)) \rightarrow \varphi(M \times$ $Q \times(-2,2))$ such that $g_{-1}^{\prime}$ is $1-1$ over $\varphi(\partial M \times Q \times[-1,1])$, $\left(g_{-1}^{\prime}\right)^{-1} \varphi(\partial M \times Q \times[-1,1])$ is a $Z$-set in $f^{-1} \varphi(M \times Q \times(-2,2))$, and $g_{-1}^{\prime}$ is $\mathscr{U}$-close to $f \mid$, for any prechosen open cover $\mathscr{U}$ of $\varphi(M \times Q \times$ $(-2,2)$ ). By choosing $\mathscr{U}$ sufficiently fine we can extend $g_{-1}^{\prime}$ to a $C E$ mapping $\tilde{g}_{-1}: X \rightarrow Y$ such that $\tilde{g}_{-1}=f$ over $Y \backslash \varphi(M \times Q \times(-2,2))$ and $\tilde{g}_{-1} \simeq f$ rel $X \backslash f^{-1} \varphi(M \times Q \times(-2,2))$. The collaring theorem of [3], which asserts that every $Q$-manifold which is a $Z$-set in another $Q$-manifold is also collared in that $Q$-manifold, implies that $\tilde{g}_{-1}^{-1} \varphi(\partial M \times$ $Q \times[-1,1])$ has a collar neighborhood in $X$. That is, there exists an open embedding

$$
\alpha: \tilde{g}_{-1}^{-1} \varphi(\partial M \times Q \times[-1,1]) \times[0,1) \rightarrow X
$$

such that $\alpha(x, 0)=x$, for all $x \in \tilde{g}_{-1}^{-1} \varphi(\partial M \times Q \times[-1,1])$. It is also true that $\partial M \times[-1,1]$ has a collar neighborhood in $M \times(-2,2)$ which contains $M_{-1} \times[-1,1]$. This is a finite-dimensional problem and uses the fact that $M_{-1}$ is a regular neighborhood of $\partial M$. Thus $\varphi(\partial M \times Q \times[-1,1])$ has a collar neighborhood in $\varphi(M \times Q \times$ $(-2,2))$ which contains $\varphi\left(M_{-1} \times Q \times[-1,1]\right)$. Using these collar neighborhoods it is easy to modify $\tilde{g}_{-1}$ to get our desired $g_{-1}$.

To construct $g_{i}, 0 \leqq i \leqq n$, we just inductively work our way through the handles of the decomposition, applying Lemma 2 repeatedly. We leave the details to the reader.

Finally we will need a relative version of Theorem 1 for the compact case.

LEMMA 4. Let $X, Y$ be compact $Q$-manifolds, $A \subset Y$ be a $Z$-set, and let $f: X \rightarrow Y$ be a $C E$ mapping such that $f$ is $1-1$ over $A$ and $f^{-1}(A)$ is a Z-set in $X$. Then there exists a homeomorphism $g: X \rightarrow Y$ such that $g=f$ on $f^{-1}(A)$ and $g \simeq f \operatorname{rel} f^{-1}(A)$. 
Proof. The triangulation theorem of [4] asserts that $Y$ is homeomorphic to $\mathrm{K} \times Q$, for some finite simplicial complex $K$. The comments made at the beginning of the proof of Lemma 3 imply that $Y$ is homeomorphic to $M \times Q$, for some compact, combinatorial, $n$-manifold $M$. Thus $Y$ can be replaced by $M \times Q$ and the main theorem of [2] concerning $Z$-sets in $Q$-manifolds implies that we can assume $A \subset \partial M \times Q$. [The main theorem of [3] implies that there is a homeomorphism $h$ of $M \times Q$ onto $M \times[0,1] \times Q$ which takes $A$ into $M \times\{0\} \times Q$. Then $h(A) \subset$ $\partial\left(M^{\prime}\right) \times Q$, where $\left.M^{\prime}=M \times[0,1].\right]$

Using Lemma 1 let $\tilde{g}_{-1}: X \rightarrow M \times Q$ be a $C E$ mapping such that $\tilde{g}_{-1}$ is $1-1$ over $\partial M \times Q, \tilde{g}_{-1}^{-1}(\partial M \times Q)$ is a $Z$-set in $X, \tilde{g}_{-1}$ is $\mathscr{U}$-close to $f$, for any prechosen open cover $\mathscr{U}$ of $Y$, and $\tilde{g}_{-1}=f$ over $A$. [To see this we apply Lemma 1 to the restricted $C E$ mapping $f \mid: X \backslash f^{-1}(A) \rightarrow$ $(M \times Q) \backslash A$.] If $\mathscr{U}$ is sufficiently fine, then we have $\tilde{g}_{-1} \simeq f \operatorname{rel} f^{-1}(A)$.

As in the proof of Lemma 3 let $M=M_{-1} \cup M_{0} \cup \cdots \cup M_{n}$ be a $P L$ handle decomposition of $M$ and modify $\tilde{g}_{-1}$ to get a $C E$ mapping $g_{-1}: X \rightarrow Y$ such that $g_{-1}$ is 1-1 over a neighborhood of $M_{-1} \times Q$, $g_{-1}=\tilde{g}_{-1}$ over $\partial M \times Q$, and $g_{-1} \simeq \tilde{g}_{-1} \operatorname{rel} \tilde{g}_{-1}^{-1}(\partial M \times Q)$. Then we inductively work our way through the handles in a standard manner, applying Lemma 2 at each step.

3. Proof of Theorem 1. In Lemma 4 we treated the compact case so let us assume that we have a $C E$ mapping $f: X \rightarrow Y$, where $X$ and $Y$ are noncompact $Q$-manifolds. The triangulation theorem of [5] implies that we can replace $Y$ by $K \times Q$, where $K$ is a countable, locally-finite, simplicial complex. Write $K=\bigcup_{n=1}^{\infty} K_{n}$, where each $K_{n}$ is a finite subcomplex of $K$ such that $K_{n} \subset \operatorname{Int}\left(K_{n+1}\right)$ and $\operatorname{Bd}\left(K_{n}\right)$ is a finite subcomplex which is $P L$ bicollared in $K$. [To achieve this we might have to subdivide $K$.] Thus for each $n \geqq 1$ we have an open embedding

$$
\varphi_{n}: \operatorname{Bd}\left(K_{n}\right) \times Q \times R \rightarrow\left(\operatorname{Int}\left(K_{n+1}\right) \backslash K_{n-1}\right) \times Q
$$

such that $\varphi_{n}(x, q, 0)=(x, q)$, for all $(x, q) \in \operatorname{Bd}\left(K_{n}\right) \times Q$. Moreover the $\varphi_{n}$ 's can be chosen so that their images are pairwise disjoint.

It follows from Lemma 3 that there exists a $C E$ mapping $g_{1}: X \rightarrow Y$ such that

$$
\begin{gathered}
g_{1}=f \text { over }(K \times Q) \backslash \bigcup_{n=1}^{\infty} \varphi_{n}\left(\operatorname{Bd}\left(K_{n}\right) \times Q \times(-2,2)\right), \\
g_{1} \text { is } 1-1 \quad \text { over } \bigcup_{n=1}^{\infty} \varphi_{n}\left(\operatorname{Bd}\left(K_{n}\right) \times Q \times[-1,1]\right),
\end{gathered}
$$

and $g_{1}$ is proper homotopic to $f$. Now consider the restricted $C E$ mapping 
$g_{1} \mid: X_{1} \rightarrow Y_{1}$, where

$$
Y_{1}=(K \times Q) \backslash \bigcup_{n=1}^{\infty} \varphi_{n}\left(\operatorname{Bd}\left(K_{n}\right) \times Q \times\left(-\frac{1}{2}, \frac{1}{2}\right)\right) \text { and } X_{1}=g_{1}^{-1}\left(Y_{1}\right)
$$

Note that $Y_{1}$ is the union of compact $Q$-manifolds which are pairwise disjoint. Moreover the topological boundaries of these compact $Q$ manifolds (i.e. boundaries in $K \times Q$ ) are $Z$-sets in $Y_{1}$ such that $g_{1} \mid$ is 1-1 over each one and the inverse image of each one under $g_{1} \mid$ is a $Z$-set in $X_{1}$. Thus Lemma 4, applied to these compact $Q$-manifolds, gives a homeomorphism $\tilde{g}_{2}: X_{1} \rightarrow Y_{1}$ which extends to a homeomorphism $g_{2}: X \rightarrow Y$ such that $g_{2}=g_{1}$ over $\bigcup_{n=1}^{\infty} \varphi_{n}\left(\operatorname{Bd}\left(K_{n}\right) \times Q \times\left[-\frac{1}{2}, \frac{1}{2}\right]\right)$ and $g_{2}$ is proper homotopic to $g_{1}$. Thus $g_{2}$ is our required homeomorphism.

4. Proof of Theorem 2. In [6] it was shown that if $K, L$ are strongly locally-finite CW-complexes and $f: K \rightarrow L$ is a proper homotopy equivalence, then $f$ is an infinite simple homotopy equivalence if $f \times$ id: $K \times$ $Q \rightarrow L \times Q$ is proper homotopic to a homeomorphism of $K \times Q$ onto $L \times Q$.

If $f: K \rightarrow L$ is a $C E$ mapping, then $f \times$ id: $K \times Q \rightarrow L \times Q$ is also a $C E$ mapping. It follows from [12] that $K \times Q$ and $L \times Q$ are $Q$-manifolds. Thus Theorem 2 follows from Theorem 1.

5. Open questions. We list here two questions which are related to Theorem 2 but which do not appear to be susceptible to the same techniques. In what follows let $A$ be a compact ANR and let $K, L$ be finite CW-complexes.

Question 1. If $f: K \rightarrow A, g: L \rightarrow A$ are $C E$ mappings, then does there exist a simple homotopy equivalence $h: K \rightarrow L$ such that $g h \simeq f$ ?

Question 2. If $f: A \rightarrow K, g: A \rightarrow L$ are $C E$ mappings, then does there exist a simple homotopy equivalence $h: K \rightarrow L$ such that $h f \simeq g$ ?

\section{REFERENCES}

1. K. Borsuk, Concerning homotopy properties of compacta, Fund. Math. 62 (1968), 223-254. MR 37 \#4811.

2. T. A. Chapman, Dense sigma-compact subsets of infinite-dimensional manifolds, Trans. Amer. Math. Soc. 154 (1971), 399-426. MR 44 \# 1058.

3. - On the structure of Hilbert cube manifolds, Compositio Math. 24 (1972), 329-353.

4. - Compact Hilbert cube manifolds and the invariance of Whitehead torsion, Bull. Amer. Math. Soc. 79 (1973), 52-56.

5. - All Hilbert cube manifolds are triangulable (preprint).

6. - Classification of Hilbert cube manifolds and infinite simple homotopy types (preprint).

7. - - Cell-like mappings of Hilbert cube manifolds: Solution of a handle problem (in preparation). 
8. M. Cohen, Simplicial structures and transverse cellularity, Ann. of Math. (2) 85 (1967), 218-245. MR 35 \# 1037.

9. L. C. Siebenmann, Infinite simple homotopy types, Nederl. Akad. Wetensch. Proc. Ser. A 73 = Indag. Math. 32 (1970), 479-495. MR 44 \#4746.

10. - Approximating cellular maps by homeomorphisms, Topology 11 (1972), 271-294. MR 45 \#4431.

11. J. E. West, Infinite products which are Hilbert cubes, Trans. Amer. Math. Soc. 150 (1970), 1-25. MR 42 \#1055.

12. - Mapping cylinders of Hilbert cube factors, General Topology and Appl. 1 (1971), no. 2, 111-125. MR 44 \# 5984.

Department of Mathematics, University of Kentucky, LeXington, Kentucky 40506 